\title{
Simultaneous Determination of Protein Backbone Structure and Dynamics from Residual Dipolar Couplings
}

\author{
Supporting Information
}

\section{Dynamic meccano procedure.}

\author{
Alignment Tensor Determination
}

This step consists in determining, simultaneously, the components $\left\{D_{a}, D_{r}, \alpha, \beta, \gamma\right\}$ of all tensors (without knowing the structure) and the dynamic amplitude for each site associated with the appropriate dynamic model that should be identified during the procedure.

This entails the determination of 22 independent parameters defining the tensors (five values of $D_{a}$ and $D_{r}$, and the relative orientation of the two tensors $\alpha, \beta, \gamma\}$, and four parameters defining the orientation of each considered plane and its associated dynamic amplitude. A total of $(4 n+22)$ for the tensor that allows for the presence of dynamics, and $(3 n+22)$ for the tensor that is determined without allowing for local dynamics.

Weighting of the 15 different datasets was determined in all cases using a robust Rousseeuw and Croux scale estimator (P.J. Rousseeuw, C. Croux, J. Amer. Statistical Assoc. 1993, 88, 1273-1283).

The algorithm proceeds as follows:

We run a first fitting cycle in which the eigenvalues of all tensors, the orientation of each peptide plane, the type of dynamic model and the amplitude parameter associated with it, are simultaneously optimized.

The peptide plane conformation is assumed to be the same throughout the molecule, and defined from a database of high-resolution crystal structures (see below)

The dynamic parameter corresponds to the amplitude of anisotropic peptide plane motion (one dimensional Gaussian axial fluctuation or 1D-GAF) about three orthogonal axes $\left\{\sigma_{\alpha}, \sigma_{\beta}\right.$ or $\left.\sigma_{\gamma}\right\}$ or a simple scaling factor for each coupling $\{S\}$.

Averaging of RDCs under the latter motion requires only scaling of a single order parameter S common to all RDCs in the plane, while the three anisotropic models affect differently oriented vectors in a different way. GAF motions are introduced analytically, using expressions given below. The most appropriate of these motions is retained on the basis of the fitting function $\chi^{2}$.

\section{Structure Determination}

Following determination of the components of the alignment tensor, the algorithm constructs the protein backbone by sequential positioning of peptide planes and intervening tetrahedral junctions.

The initial plane orientation (C-terminal plane) is determined by minimising a target function containing all RDCs in the plane. This orientation is defined with a degeneracy of 2 , so that from this point two entire calculations are performed.

Subsequent cycles sequentially determine the orientation of plane (i) with respect to plane (i+1) by minimising a target function containing all RDCs in the plane, the RDCs in the intervening tetrahedral junction, and the value of the tetrahedral junction angle (mean assumed to be $111^{\circ}$ ). In order to allow for experimental outliers a robust maximum-likelihood estimator was used in the place of a classical $\chi^{2}$ function.

1D-GAF Formula

The effect of a 1D-GAF motion on a residual dipolar coupling can be analytically expressed as follows: 


$$
\begin{aligned}
& \left.\left\langle D_{i j}\right\rangle_{1 D-G A F}=-\frac{\mu_{0}}{8 \pi^{2}} \frac{\gamma_{i} \gamma_{j} \hbar}{r_{i j}^{3}}\left\{\begin{array}{l}
\frac{1}{4} D_{a}\left[S_{1}\left(3 \cos ^{2} \beta-1\right)+3 s_{2} \sin 2 \beta \cos \alpha+3 s_{3} \sin ^{2} \beta \cos 2 \alpha\right. \\
\frac{3}{8} D_{r}\left[\begin{array}{l}
s_{1} \sin ^{2} \beta \cos 2 \gamma- \\
2 s_{2} \sin \beta\left(\cos (\alpha+2 \gamma) \cos ^{2} \frac{\beta}{2}-\cos (\alpha-2 \gamma) \sin ^{2} \frac{\beta}{2}\right) \\
2 s_{3}\left(\cos (2 \alpha+2 \gamma) \cos ^{4} \frac{\beta}{2}+\cos (2 \alpha-2 \gamma) \sin ^{4} \frac{\beta}{2}\right)
\end{array}\right]
\end{array}\right]\right\} \\
& \text { with } s_{1}=2\left(3 \cos ^{2} \theta-1\right) ; s_{2}=2 \sin 2 \theta \exp \left(-\frac{\sigma^{2}}{2}\right), s_{2}=2 \sin ^{2} \phi \exp \left(-2 \sigma^{2}\right)
\end{aligned}
$$

where $r_{i j}$ is the internuclear distance, $\sigma$ the motional amplitude, $\{\alpha, \beta, \gamma\}$ the Euler angles transforming from the alignment tensor principal axis system into the local peptide plane frame defined by its $z$-axis along the rotational axis and its $x$-axis so that the internuclear vector lies in the $\mathrm{x}-\mathrm{z}$-plane and $\theta$ the angle between the rotational axis and the internuclear vector.

\section{Peptide plane Structure :}

CA1 : $\{0.000,0.000,0.000\}$

$C^{\prime}:\{1.430,-0.529,0.000\}$

$\mathrm{N}:\{2.404,0.382,0.000\}$

$\mathrm{HN}:\{2.161,1.373,0.000\}$

CA2 $:\{3.806,-0.006,0.000\}$

Note that the fitting of dynamic parameters from structures $1 \mathrm{p} 7 \mathrm{e}$ and 1igd uses the heavy atom geometry of the structures, and not perfect peptide plane geometry.

\section{Q-values for CaHa Reproduction (all residues taken into consideration):}

These are effectively static values, with no differential dynamic scaling for the different sites in either case.

$\begin{array}{llllll} & \text { Bicelles } & \text { PEG } & \text { Phage } & \text { NCPG } & \text { PCPG } \\ \text { Dynamic meccano: } & 10.1 & 10.0 & 11.8 & 19.2 & 12.9 \\ \text { X-ray Structure } \quad: & 12.1 & 11.9 & 22.7 & 23.9 & 15.8\end{array}$

\section{Sidechain conformation :}

The meccano structure determination only describes the positions of backbone atoms (including therefore the beta carbons), but not the side-chains. We can therefore only speculate on the positions of sidechains for the meccano conformation. Side-chains can be added by using a predictive method available via the program 'SCCOMP', developed by Eyal et al (Eyal, E., Najmanovich, R., McConkey, B. J., Edelman, M. \& Sobolev, V. (2004) J. Comp. Chem., 25, 712-724) that uses a scoring function based on surface areas between atoms and solvent accessible surface. This results in a very similar conformation to that found in the X-ray structure (heavy atom rmsd for residues $8-60=0.94 \AA$ ). This result is in line with previous observations of prediction of side-chain conformations on the basis of known backbone conformation. 
Comparison of order parameters derived from spin relaxation (black) with those determined from the 3D GAF analysis of the dynamic meccano structure (red).

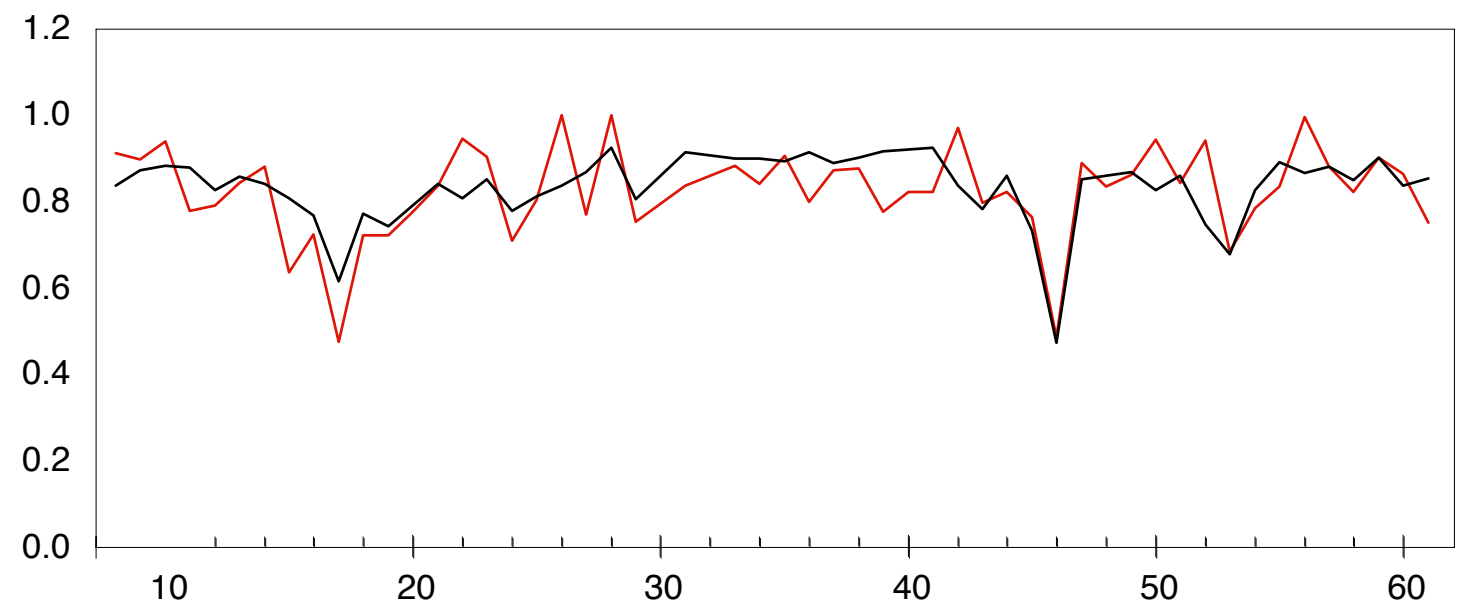

